\title{
INVENTÁRIO DO SAPATEIRO DAMIÃO SIMÕES: UM ESTUDO FÍSICO-QUÍMICO DO DOCUMENTO MAIS ANTIGO DO ESTADO DE SÃO PAULO
}

\author{
Maria T. C. Sansiviero ${ }^{\mathrm{a}, *,(\mathbb{C}, \text { Carlos E. Sampietri }}{ }^{\mathrm{b}}$, Marcos V. da Costa ${ }^{\mathrm{b}}$, Norma C. Cassares ${ }^{\mathrm{b}}$ e Dalva L. A. Faria ${ }^{\mathrm{a}}$ \\ aDepartamento de Química Fundamental, Instituto de Química, Universidade de São Paulo, 05508-000 São Paulo - SP, Brasil

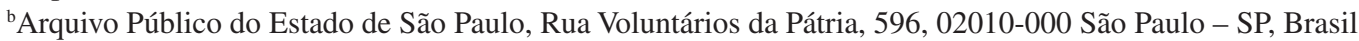

Recebido em 04/06/2019; aceito em 28/08/2019; publicado na web em 21/10/2019

\begin{abstract}
THE INVENTORY OF THE SHOEMAKER DAMIÃO SIMÕES: A PHYSICO-CHEMICAL STUDY OF THE MOST ANCIENT DOCUMENT OF SÃO PAULO STATE. The oldest manuscript from the Arquivo Público do Estado de São Paulo dating back to the XVI century, which was laminated some decades ago, was analyzed by Raman and FTIR spectroscopies. A book from the XVIII century and Japanese papers were also investigated for comparison purpose. Raman microscopy is a well-known technique, which offers a non-destructive approach to informing the preservation state of paper and textile heritage. The manuscript and book were both characterized as cotton rag paper and their degradation state were evaluated from the spectroscopic features. The use of certain Raman signatures as ageing markers was discussed and questioned when different types of cellulose or even processing are compared.
\end{abstract}

Keywords: rag paper; paper degradation; Raman spectroscopy; cultural heritage conservation.

\section{INTRODUÇÃO}

O diagnóstico de manuscritos com suporte em papel é uma questão importante relacionada com a conservação e restauração de documentos antigos. ${ }^{1} \mathrm{O}$ papel usado para escrita chegou à Europa em torno do século XII vindo da China; ele era então obtido de trapos de algodão, linho ou cânhamo, ${ }^{1,2}$ em vez de polpa de madeira que foi introduzida somente a partir de meados do século XIX. ${ }^{2} \mathrm{O}$ papel de trapo (rag paper) é um feltro de fibras de celulose, com adição de materiais de preenchimento.

Quimicamente, o papel consiste principalmente de fibras de celulose ligadas, ou seja, polímeros lineares de monômeros de glucose ( $\beta$-D-glucopiranose) unidos por ligações $\beta$-1,4 glicosídicas (Figura 1). O número de monômeros ligados (grau de polimerização) varia dependendo da fonte da celulose, por exemplo, para celulose de madeira tem-se um grau de polimerização de até $10.000\left(3,4 \times 10^{6} \mathrm{~g} \mathrm{~mol}^{-1}\right)$ enquanto na celulose de algodão o grau de polimerização é de 15.000 $\left(5,1 \times 10^{6} \mathrm{~g} \mathrm{~mol}^{-1}\right)$. As cadeias se mantêm unidas por fortes ligações de hidrogênio intermoleculares que são responsáveis pela estrutura altamente orientada que apresentam. ${ }^{3}$ Moléculas de água também estão presentes e desempenham um papel muito importante nas ligações de hidrogênio, uma vez que a celulose é naturalmente hidrofílica.

A cadeia polimérica da celulose pode ser degradada por hidrólise e/ou oxidação. ${ }^{4}$ Um problema fundamental na conservação e restauro é a identificação e compreensão dos mecanismos de degradação da celulose, usando para isso, sempre que possível, métodos não destrutivos como a espectroscopia de espalhamento Raman e de absorção no infravermelho. Os benefícios da espectroscopia Raman, ou mais especificamente da microscopia Raman, são o fato de permitir análises não destrutivas e sondar áreas muito pequenas de amostras, bem como geralmente dispensar qualquer tipo de manipulação da amostra. Por essa razão tem sido empregada na investigação do envelhecimento de papéis.

Neste trabalho, microscopia Raman e FTIR foram utilizadas na identificação do tipo de celulose e do estado de degradação do papel que foi utilizado em um manuscrito do século XVI. O documento em questão é o Inventário do Sapateiro Damião Simões (1578), o mais antigo do Arquivo Público do Estado de São Paulo. O documento é mostrado na Figura 2, na qual é possível notar que o papel está amarelado e danificado, especialmente nas bordas. Além disso, há algumas décadas o documento sofreu um processo de laminação (plastificação), que consiste na aplicação em ambos os lados do documento de um filme termoplástico, na tentativa de preservá-lo; essa prática foi empregada nas décadas de 1950 e 1960, porém hoje em dia esse processo não é mais utilizado.

O material usado na plastificação do manuscrito foi também caracterizado visando avaliar a possibilidade de sua possível remoção. Nesse fragmento do passado, com 32 páginas, é possível conhecer

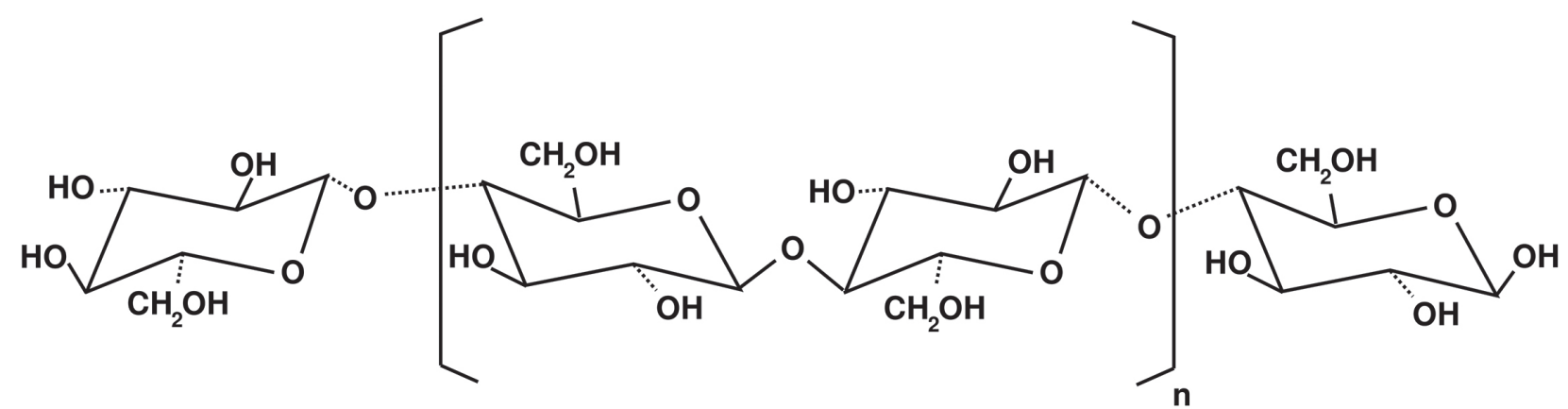

Figura 1. Estrutura molecular da celulose baseada no monômero $\alpha$-D-glucose com unidades $\beta$-1,4-glicosídicas ligadas 
detalhes da vida naquela época remota. Damião Simões foi um sapateiro que viveu na São Paulo primitiva, do século 16. O processo de Damião, aberto em 1578, quando de sua morte, foi encerrado quando o filho, também Damião, após ter completado a idade exigida para poder manejar os objetos, em 1602, recebeu os pertences do pai, que estavam sob a guarda do juiz. Esse documento pertence a um conjunto documental chamado de Inventários e Testamentos, provenientes dos cartórios dos antigos juizados de órfãos que, pela lei no 666 de 1899, foram recolhidos ao Arquivo Público do Estado na primeira década do século XX. O Arquivo Público do Estado de São Paulo, cumpre lembrar, foi instituído em 1892, logo após a proclamação da República, para abrigar documentos, mapas e memórias de importância histórica para o Estado.

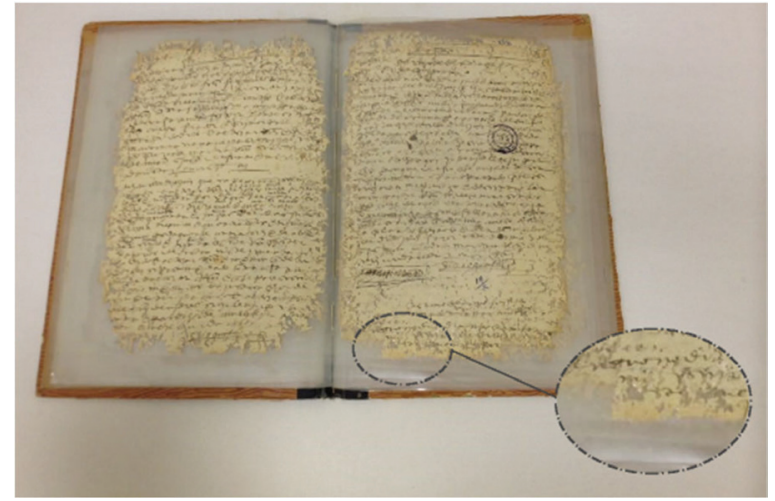

Figura 2. Inventário do Sapateiro Damião Simões - manuscrito de 1578

Para fins de comparação com os dados obtidos para o manuscrito de 1578 foi utilizado um livro de 1777, que também emprega papel de trapos, considerando-se a época em que o livro foi publicado. Papéis japoneses ${ }^{5}$ também foram analisados, principalmente tendo em conta sua importância no restauro de documentos antigos e porque são papéis neutros e sem lignina.

\section{PARTE EXPERIMENTAL}

\section{Material}

O manuscrito, doravante identificado como Inventário, encontra-se plastificado em matriz polimérica transparente e, apesar de ser possível estudá-lo na forma em que se encontra, foi também retirado um pequeno fragmento $\left(c a .8 \mathrm{~mm}^{2}\right)$ da borda do documento que não tinha texto escrito, de onde foram feitas análises estratigráficas por microscopia Raman tanto do envoltório termoplástico quanto do papel. Desse fragmento também foram cuidadosamente retiradas fibras para que fossem analisadas por microscopia de absorção no infravermelho (Fourier Transform Infrared, FTIR), FT-Raman e Microscopia Eletrônica de Varredura (Scanning Electron Microscopy, SEM).

Pequenos fragmentos ( $c a .5 \mathrm{~mm} \times 5 \mathrm{~mm}$ ) das bordas de diferentes páginas de um livro datado de 1777 (escrito em latim e impresso em Veneza, intitulado Teologia, de autoria de Fungentio Cuniliati e B. Jacobi Salomoni) foram selecionados para efetuar as análises de espectroscopia FT-Raman e microscopia FTIR; tais amostras serão identificadas aqui como Livro.

Algumas amostras de papel japonês (Washi), que consiste em papéis artesanais feitos com as cascas dos arbustos Gampi (Wilstroemia sikokiana), Kozo (Broussonetia kazinoki) e Mitsumata (Edgeworthia chrysantha) foram também analisadas para fins de comparação, por não conterem lignina, possuírem fibras de tamanhos variados e terem sido processados de diferentes formas. Esses papéis são contemporâneos e estão identificados neste texto como Gampi (cozimento das fibras com carbonato de sódio e secagem sobre placa de madeira, $18 \mathrm{~g} \mathrm{~m}^{-2}$ ), Kozo (cozimento com carbonato de sódio e secagem em tambor de aço inoxidável, $18 \mathrm{~g} \mathrm{~m}^{-2}$ ), Tengujo (fibras de Kozo com cozimento em cal e secagem em tambor de aço, $11 \mathrm{~g} \mathrm{~m}^{-2}$ ) e Mitsumata (cozimento com cal e secagem em placa de madeira, $38 \mathrm{~g} \mathrm{~m}^{-2}$ ). As fibras desses papéis têm características diferentes: os papéis Gampi e Mitsumata tem fibras estreitas e relativamente curtas enquanto no papel Kozo elas são largas e bastante longas. ${ }^{6}$

Para fins de comparação também foi analisado papel sulfite contemporâneo $\left(75 \mathrm{~g} \mathrm{~m}^{-2}\right)$; esse tipo de papel é constituído por fibras curtas de celulose que são liberadas empregando uma solução ácida contendo íons sulfito e hidrogenossulfito.?

O tingimento das fibras foi feito usando o reagente de Herzberg: foi preparada uma solução saturada de cloreto de zinco em água deionizada e outra contendo iodo $(0,25 \mathrm{~g})$ e iodeto de potássio $(5,25 \mathrm{~g})$ então $25 \mathrm{~mL}$ da primeira foram misturados a $12,5 \mathrm{~mL}$ da segunda.

\section{Métodos}

A caracterização dos papéis e fibras foi feita através de espectroscopia de absorção no infravermelho por transformada de Fourier (FTIR), espectroscopia FT- Raman e microscopia Raman.

No caso do FTIR utilizou-se um espectrômetro Alpha (Bruker) com detector DTGS, acoplado a um acessório de ATR (cristal de diamante) de uma única reflexão (Platinum) e também um microscópio (Lumos, Bruker) com detector MCT refrigerado com nitrogênio líquido e objetiva de ATR de germânio.

Os espectros Raman foram obtidos em equipamento Renishaw inVia refrigerado termoeletricamente $\left(-70^{\circ} \mathrm{C}\right)$ e que utiliza um microscópio Leica DM2500 M e lente objetiva de 50 vezes (NA 0,75); a radiação laser empregada (laser de estado sólido, Renishaw) foi a linha em $785 \mathrm{~nm}$ e os espectros foram obtidos com rede de difração de 1200 linhas $\mathrm{mm}^{-1}$. Com este equipamento foi possível realizar análise estratigráfica do fragmento removido do Inventário; para isso foi feito um corte transversal na amostra, expondo as diversas camadas que a constituíam (polímeros e papel) e essa amostra foi montada perpendicularmente em um suporte, o que permitiu a análise individual de cada camada. Um equipamento FT-Raman (RFS 100/S, Bruker) foi empregado no caso dos espectros obtidos no infravermelho próximo (1064 nm, laser de $\mathrm{Nd}^{3+} / \mathrm{YAG}$ ); neste caso a resolução espectral foi $4 \mathrm{~cm}^{-1}$. A relação de intensidades Raman foi obtida através da altura das bandas considerando que não houve alteração nem em largura nem em formato das bandas para diferentes amostras. Na obtenção das intensidades foi utilizado o programa Grams AI (Thermo) com correção da linha base por múltiplos pontos, mantendo-se os parâmetros do ajuste de bandas para todas as amostras. Em todos os casos os espectros foram registrados em diferentes pontos de uma mesma amostra e em diferentes amostras; em alguns casos optou-se por reportá-los como espectros médios para melhorar a relação sinal/ruído.

O equipamento usado para a microscopia eletrônica de varredura foi um JEOL JSM-7401F $(5,0 \mathrm{kV}, 10 \mu \mathrm{A}$, distância de trabalho de $8,1 \mathrm{~mm}$ e detector de elétrons secundários (LEI)); a amostra foi colocada sobre fita de cobre e recoberta com Au (10 nm). As fibras tingidas foram observadas e fotografadas empregando um estereoscópio Nikon SMZ645 acoplado a um iluminador de fibra ótica dupla (Motic MLC-150C).

Cálculos DFT foram realizados para a molécula de celobiose $\left(\mathrm{C}_{12} \mathrm{H}_{22} \mathrm{O}_{11}\right)$ usando o algoritmo B3LYP (Becke, Lee, Yang e Parr) e base 6-31G* (Spartan 18, Wavefunction); essa molécula foi escolhida por ser um dissacarídeo constituído por duas moléculas de glicose unidas por ligação $\beta$-1,4-glicosídica sendo, portanto, a unidade que se repete na celulose. 


\section{RESULTADOS E DISCUSSÃO}

Como já foi dito anteriormente, o documento de 1578 (Inventário) teve suas páginas plastificadas, uma prática comum nos anos 1950 e $1960^{8}$ que tinha por objetivo melhorar a aparência e proteger a folha impressa por aumentar a resistência ao atrito e servir como barreira à umidade, à gordura etc. $\mathrm{O}$ processo de plastificação ou laminação mais comum aplica, sob pressão e calor, um filme de polietileno (PE) com espessura de 12 a $15 \mu \mathrm{m}$ sobre o documento; para impedir a aderência do filme de polietileno ao cilindro térmico usa-se, como agente desmoldante, uma película de poliéster (poli (tereftalato de etileno), PET) que possui alta temperatura de fusão $\left(260{ }^{\circ} \mathrm{C}\right)$ na espessura de 18 a 23 um. $^{9}$

O método utilizado atualmente pelo Arquivo Público do Estado de São Paulo na restauração de documentos históricos com suporte em papel emprega papel japonês, ${ }^{6}$ o que tornaria necessária a remoção da matriz polimérica. Nesse sentido, a possibilidade de remoção foi investigada inicialmente por meio da caracterização dos polímeros a fim de verificar se tem a mesma composição química dos produtos comerciais atualmente no mercado. Para isso foram utilizadas as espectroscopias FTIR e microscopia Raman; nesse último caso, a alta resolução espacial (poucos $\mu \mathrm{m}^{2}$ ) permitiu a análise das distintas camadas observadas em um corte transversal (corte estratigráfico) de um pequeno fragmento de amostra proveniente do Inventário.

A Figura 3 mostra os espectros FTIR do fragmento extraído do Inventário; é possível ver que há bandas de dois polímeros distintos e apesar de se poder distinguir pelo perfil espectral que se tratava de PE e PET, aqueceu-se ligeiramente a amostra até que fosse possível remover uma pequena porção do polímero mais interno (em contato direto com o papel) e o espectro FTIR obtido (Figura 3, PE) confirmou que se tratava de polietileno, com bandas características ${ }^{10} \mathrm{em} 1067$ e $1128 \mathrm{~cm}^{-1}(\mathrm{v}(\mathrm{CC})), 1298$ e $1458 \mathrm{~cm}^{-1}\left(\delta\left(\mathrm{CH}_{2}\right)\right)$ e 2847 e $2886 \mathrm{~cm}^{-1}$ $(\mathrm{v}(\mathrm{CH}))$. A análise do corte estratigráfico do fragmento removido do Inventário feita por microscopia Raman (Figura 4) confirmou que

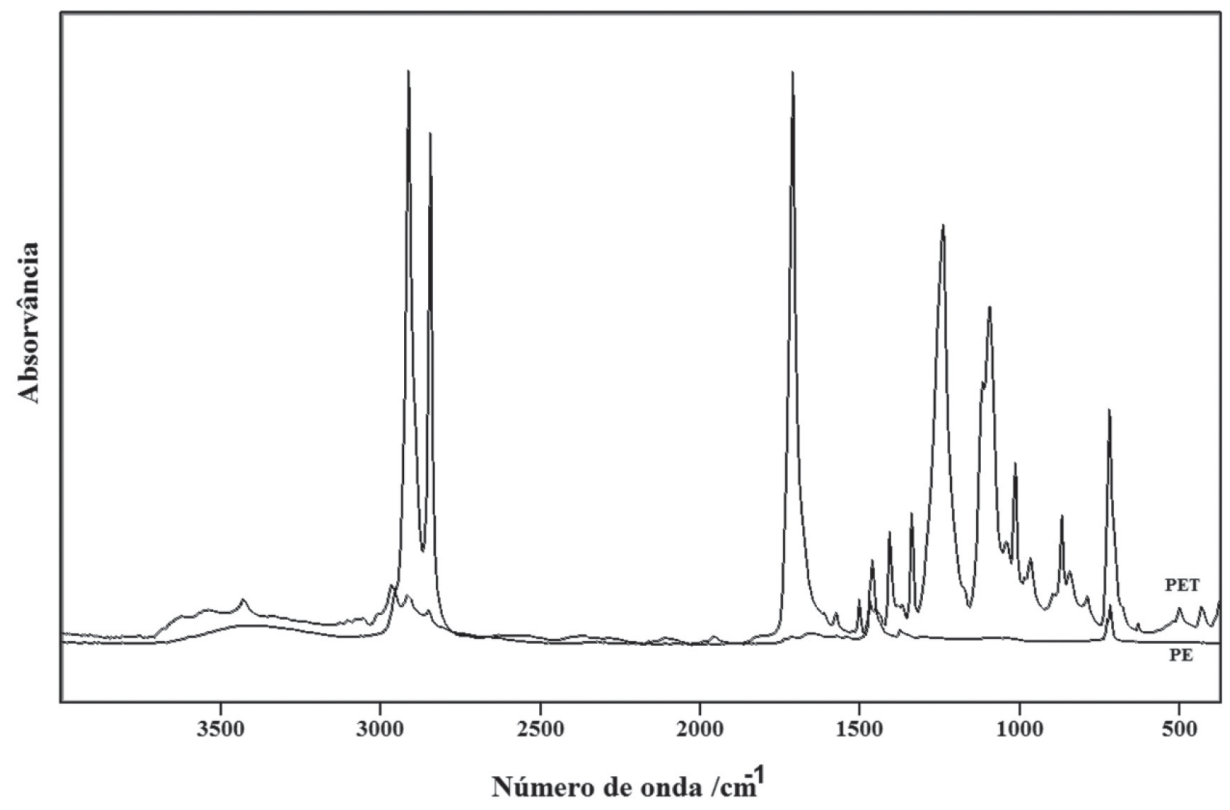

Figura 3. Espectros FTIR dos polímeros polietileno (PE) e poli (tereftalato de etileno) (PET) usados na laminação do Inventário

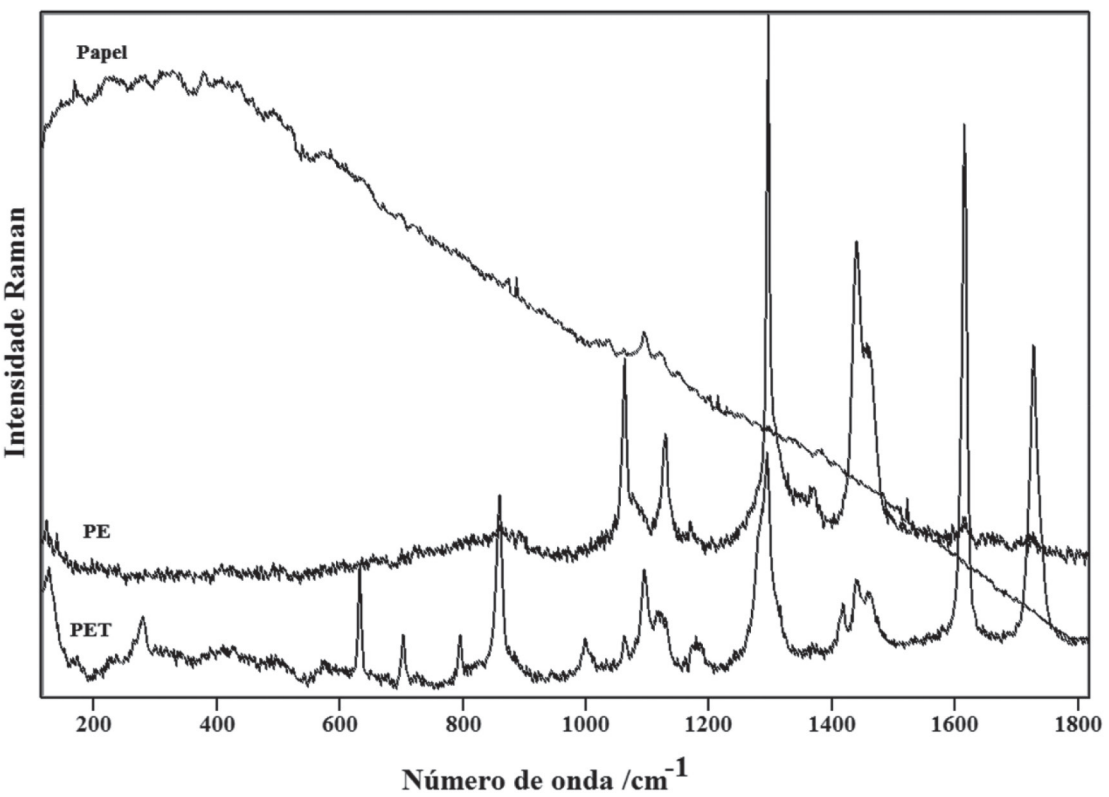

Figura 4. Espectros obtidos por Microscopia Raman $(785 \mathrm{~nm})$ de corte estratigráfico de uma amostra do Inventário 
o polímero em contato com o cilindro aquecido era poli (tereftalato de etileno), o qual apresenta bandas em $1615 \mathrm{~cm}^{-1}$ ( $\mathrm{v}(\mathrm{CC})$ do anel benzênico) e $1728 \mathrm{~cm}^{-1}$ ( $\left.\mathrm{v}(\mathrm{CO})\right)$; a microscopia Raman confirmou também a identidade do polímero mais interno como sendo PE.

Considerando a natureza química do filme plastificante e a resistência térmica e química do PET, tentativas para sua remoção feitas pela equipe do Arquivo Público de São Paulo empregando calor e solventes foram infrutíferas. Infelizmente, na plastificação o polietileno infiltrou-se nas fibras do papel, que já estavam danificadas pela ação do tempo, tornando impossível sua remoção sem que houvesse um risco imenso para o documento.

Apesar de não ser possível remover de modo seguro os polímeros usados na plastificação, a caracterização do papel empregado no Inventário foi realizada uma vez que o mesmo nunca havia sido investigado e há pouquíssimos estudos dessa natureza sobre documentos históricos brasileiros. ${ }^{11,12}$ Para isso, espectroscopia vibracional foi usada e, no caso da espectroscopia Raman, a alta luminescência registrada quando se empregou a radiação em $785 \mathrm{~nm}$ (Figura 4) tornou necessária também a utilização de comprimento de onda mais longo, o que foi feito em equipamento FT-Raman (1064 nm). Com a linha em $785 \mathrm{~nm}$ também tentou-se analisar algumas fibras retiradas do fragmento do Inventário, descrito acima; como no caso da análise estratigráfica, o fundo de luminescência registrado também para as fibras encobriu as bandas Raman. Esse fato está de acordo com a literatura, segundo a qual espectros Raman de têxteis antigos degradados apresentam forte luminescência quando excitados no visível ou próximo ao visível. ${ }^{13}$ Essa luminescência pode ser causada por materiais depositados na superfície do objeto, tais como microrganismos (incluindo seus fragmentos e metabólitos), produtos de degradação das fibras, sujidades etc. ${ }^{13}$ Como mostrado na Figura 5, o uso da radiação de energia menor (1064 nm) permite contornar o problema da luminescência apresentado pelo papel usado no Inventário; espectros FT-Raman obtidos de diferentes áreas da amostra foram similares, indicando sua homogeneidade, tanto em termos de composição quanto de degradação, o mesmo ocorrendo no caso do Livro. Os espectros $\mu$-FTIR desses documentos são mostrados na Figura 6 e, analogamente ao que foi visto nos espectros FT-Raman, pela similaridade dos resultados pode-se concluir que as amostras eram homogêneas, o que dispensou o uso de procedimentos estatísticos na análise dos resultados.
Nos espectros mostrados na Figura 5 (Inventário e Livro) observam-se bandas características de celulose em 379, 435, 565, 1096, 1121, 1151, 1295, 1350, 1393 e 2900 cm$^{-1}$; como há superposição dos espectros pode-se depreender que ambos os papéis tenham sido feitos com o mesmo tipo de fibra. Devido ao fato das posições e intensidades relativas das bandas serem as mesmas, os valores estão em uma única coluna na Tabela 1 que também apresenta as respectivas atribuições de acordo com diferentes autores. Uma banda intensa aparece em $1645 \mathrm{~cm}^{-1}$ nos espectros $\mu$-FTIR dos papéis do Inventário e Livro e pode ser atribuída à presença de água.

Pela estrutura química da celulose é possível antecipar que os modos vibracionais devem estar bastante acoplados, fato confirmado por estudos prévios publicados na literatura. ${ }^{14-16}$ Isso é particularmente esperado na região espectral na qual alguns artigos da literatura ${ }^{11-13,17}$ posicionam as vibrações de estiramento simétrico e antissimétrico C-O-C da ligação glicosídica. Como esta ligação é rompida na degradação da celulose (hidrólise) torna-se uma candidata óbvia no diagnóstico do estado de tecidos (e papéis) e mesmo de seu grau de envelhecimento. Considerando as divergências na atribuição das bandas vibracionais, a Tabela 1 reporta as identificações feitas de acordo com vários autores. A atribuição das vibrações relacionadas à ligação $\beta$-1,4-glicosídica merecem destaque e, por esse motivo, a simulação dos espectros Raman e FTIR da celobiose (mero da celulose constituído por duas moléculas de glicose unidas por ligação $\beta$-1,4-glicosídica) foi feita usando DFT, não com o propósito de obter-se uma atribuição vibracional definitiva, mas sim de averiguar o grau de acoplamento dos modos nesse sistema modelo; infelizmente não foi possível obter a distribuição de energia potencial (potential energy distribution, PED) no cálculo realizado. A literatura ${ }^{11-13,17}$ atribui as bandas intensas no espectro Raman da celulose em 1121 $\mathrm{cm}^{-1}$ e $1096 \mathrm{~cm}^{-1}$ ao estiramento simétrico e antissimétrico do grupo COC glicosídico, respectivamente. Na animação do espectro Raman teórico da celobiose, essas vibrações não originam bandas intensas, estão bastante acopladas a outras vibrações (deformações angulares C-H e C-C principalmente) e o estiramento simétrico, em particular, tem uma apreciável contribuição de deformação angular da ligação C-O-C glicosídica no plano. É bastante provável que as bandas em $1096 \mathrm{~cm}^{-1}$ e $1121 \mathrm{~cm}^{-1}$ sejam melhor representadas por deformações angulares do que por estiramentos C-O-C em concordância com resultados da literatura ${ }^{15,16,18}$ e talvez isso explique o insucesso em

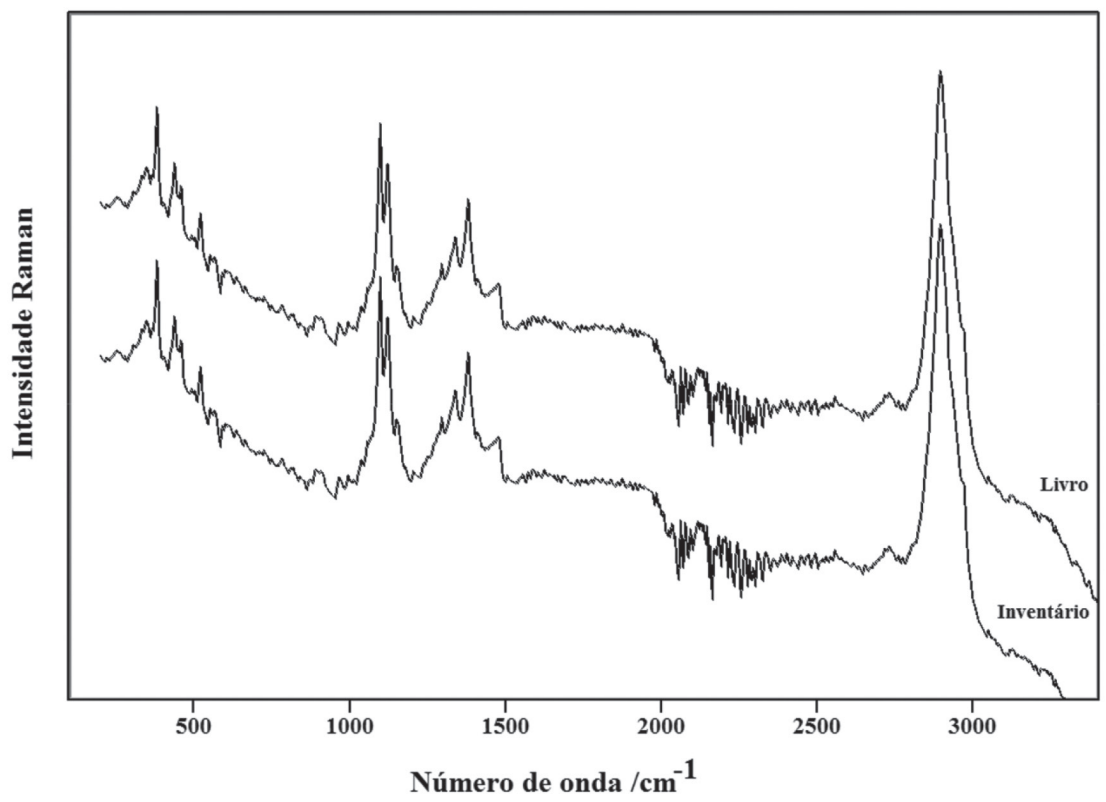

Figura 5. Espectros FT-Raman do Inventário e do Livro 


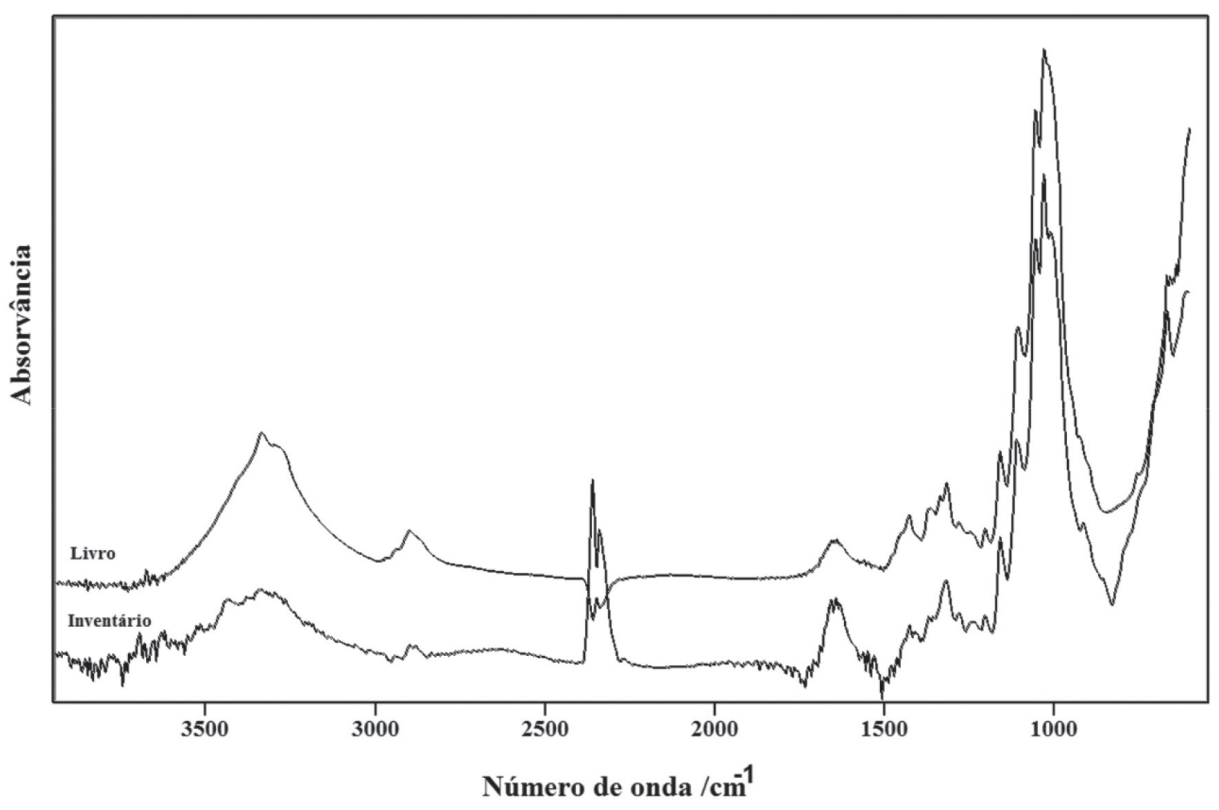

Figura 6. Espectros $\mu-$ FTIR do Inventário e do Livro

Tabela 1. Posições das bandas mais significativas observadas nos espectros Raman e de absorção no infravermelho do Inventário e do Livro, com as respectivas atribuições

\begin{tabular}{|c|c|c|c|c|}
\hline Raman $/ \mathrm{cm}^{-1}$ & $\mathrm{FTIR} / \mathrm{cm}^{-1}$ & Atribuição $0^{3,17}$ & Atribuiçãa $^{15}$ & Atribuição $^{18}$ \\
\hline & $3334 \mathrm{f}$ & $v(\mathrm{OH})$ & $v(\mathrm{OH})$ & $v(\mathrm{OH})$ \\
\hline $2970 \mathrm{~m}$ & & & as $\left(\mathrm{CH}_{2}\right)$ & $\mathrm{v}_{\mathrm{as}}\left(\mathrm{CH}_{2}\right)$ \\
\hline 2909 sh, & & $v\left(\mathrm{CH}_{2}\right)$ & $(\mathrm{CH})$ & $v(\mathrm{CH})$ \\
\hline \multirow[t]{2}{*}{$2895 \mathrm{mF}$} & $2900 \mathrm{f}$ & $v\left(\mathrm{CH}_{2}\right)$ & ${ }_{s}\left(\mathrm{CH}_{2}\right)$ & $v_{s}\left(\mathrm{CH}_{2}\right)$ \\
\hline & $1645 \mathrm{~m}$ & $\delta(\mathrm{OH})$ água & & \\
\hline $1478 \mathrm{~m}$ & & $\delta_{\mathrm{s}}\left(\mathrm{CH}_{2}\right)$ & $(\mathrm{CO}), \delta(\mathrm{HCO})$ & $\delta_{\mathrm{s}}\left(\mathrm{CH}_{2}\right)$ \\
\hline \multirow[t]{2}{*}{$1460 \mathrm{f}$} & & $\delta\left(\mathrm{CH}_{2}\right), \delta(\mathrm{COH})$ & $\delta(\mathrm{HCC}), \delta(\mathrm{HCO})$ & \\
\hline & $1425 \mathrm{~m}$ & $\delta(\mathrm{CH}) ; \delta(\mathrm{OCH})$ & $\left(\mathrm{CH}_{2}\right), \delta(\mathrm{HCC})$ & \\
\hline \multirow[t]{2}{*}{$1381 \mathrm{~m}$} & & $\rho\left(\mathrm{CH}_{2}\right)$ & $(\mathrm{HCC}), \delta(\mathrm{HCO}), \delta(\mathrm{COH})$ & $\delta(\mathrm{HCC}), \delta(\mathrm{HCO}), \delta(\mathrm{COH})$ \\
\hline & $1370 \mathrm{f}$ & $\delta(\mathrm{C}-\mathrm{H})$ & $\delta(\mathrm{HCC}), \delta(\mathrm{HCO})$ & $\delta(\mathrm{HCC}), \delta(\mathrm{HCO}), \delta(\mathrm{COH})$ \\
\hline \multirow[t]{2}{*}{$1338 \mathrm{f}$} & $1336 \mathrm{f}$ & $\omega\left(\mathrm{CH}_{2}\right) ; \delta(\mathrm{OH})$ & $(\mathrm{HCC}), \delta(\mathrm{HCO})$ & $\delta(\mathrm{HCC}), \delta(\mathrm{HCO}), \delta(\mathrm{COH})$ \\
\hline & $1316 \mathrm{~m}$ & $\delta(\mathrm{C}-\mathrm{OH}) ; \delta(\mathrm{C}-\mathrm{CH})$ & $(\mathrm{HCC}), \delta(\mathrm{HCO})$ & $\delta(\mathrm{HCC}), \delta(\mathrm{HCO}), \delta(\mathrm{COH}), \delta\left(\mathrm{CH}_{2}\right)$ \\
\hline \multirow[t]{2}{*}{$1293 \mathrm{f}$} & & $\tau\left(\mathrm{CH}_{2}\right)$ & $\delta(\mathrm{HCC}), \delta(\mathrm{HCO}), \delta(\mathrm{COH})$ & \\
\hline & $1207 \mathrm{mf}$ & $\delta(\mathrm{C}-\mathrm{H})$ & $(\mathrm{HCC}), \delta(\mathrm{HCO}), v(\mathrm{CO})$ & \\
\hline $1150 \mathrm{f}$ & $1155 \mathrm{~m}$ & $v_{\text {as }}(C C)$ anel & $(\mathrm{CO}), v(\mathrm{CC}), \delta(\mathrm{CCO}), \delta(\mathrm{COH})$ & Deformação do esqueleto \\
\hline $1121 \mathrm{~F}$ & & $v_{s}(\mathrm{COC})$ glic. & $(\mathrm{CO}), v(\mathrm{CC})$ & Deformação do esqueleto \\
\hline \multirow[t]{4}{*}{$1096 \mathrm{~F}$} & $1105 \mathrm{~m}$ & $v_{\mathrm{as}}(\mathrm{COC})$ glic. & $(\mathrm{CO}), v(\mathrm{CC}), \delta(\mathrm{COH})$ & Deformação do esqueleto \\
\hline & $1049 \mathrm{~F}$ & v(C-OH) álc. sec. & $(\mathrm{CO}), v(\mathrm{CC}), \delta(\mathrm{COH})$ & $v(\mathrm{CO}), v(\mathrm{CC})$ \\
\hline & $1034 \mathrm{~F}$ & $v(\mathrm{C}-\mathrm{OH})$ álc. prim. & $(\mathrm{CO}), v(\mathrm{CC})$ & $v(\mathrm{CO}), v(\mathrm{CC})$ \\
\hline & $917 \mathrm{f}$ & $\rho\left(\mathrm{CH}_{2}\right)$ & $(\mathrm{CO}), v(\mathrm{CC}), \delta(\mathrm{CCO}), \delta(\mathrm{HCC})$ & \\
\hline $895 \mathrm{f}$ & & $\delta_{\mathrm{s}}(\mathrm{COC})$ & $v(\mathrm{CC}), \delta(\mathrm{CCO}), \delta(\mathrm{HCC})$ & Deformação do anel \\
\hline $521 \mathrm{~m}$ & & $\delta(\mathrm{COC})$ glic. & $\delta(\mathrm{CCC}), \delta(\mathrm{CCO}), \delta(\mathrm{COC}), \delta(\mathrm{HCC})$ & \\
\hline $502 \mathrm{f}$ & & $\delta(\mathrm{COC})$ glic. & $v(\mathrm{CC}), \delta(\mathrm{CCO}), \delta(\mathrm{COC}), \delta(\mathrm{HCC})$ & \\
\hline $456 \mathrm{~m}$ & & $\delta(\mathrm{CCC})$ anel & $(\mathrm{CC}), \delta(\mathrm{CCC}), \delta(\mathrm{CCO}), \delta(\mathrm{COC})$ & \\
\hline $433 \mathrm{f}$ & & $\delta(\mathrm{CCC})$ anel & $(\mathrm{CC}), \delta(\mathrm{CCC}), \delta(\mathrm{CCO}), \delta(\mathrm{HCC})$ & \\
\hline $380 \mathrm{~m}$ & & $\delta(\mathrm{CCC})$ anel & $(\mathrm{CC}), \delta(\mathrm{CCO}), \delta(\mathrm{HCC})$ & Deformação fora do plano do anel \\
\hline $353 \mathrm{f}$ & & $\delta(\mathrm{CCC})$ anel & $(\mathrm{CO}), v(\mathrm{CC}), \delta(\mathrm{CCO})$ & \\
\hline
\end{tabular}

Abreviações: $v=$ estiramento, $\delta=$ deformação angular, $\rho=$ deformação angular antissimétrica no plano (rocking), $\tau=$ deformação angular antissimétrica fora do plano (twisting), $\omega=$ deformação angular simétrica fora do plano (wagging). Intensidades: $\mathrm{F}=$ forte, $\mathrm{f}=$ fraca, $\mathrm{m}=\mathrm{média}, \mathrm{mF}=$ muito forte, $\mathrm{mf}=$ muito fraca, $\mathrm{sh}=$ shoulder (ombro).

usar tais bandas na predição do estado de conservação de tecidos, como será visto adiante.

É importante destacar a ausência de uma banda característica de lignina em $c a .1600 \mathrm{~cm}^{-1}$ atribuída a estiramento $\mathrm{C}=\mathrm{C}$ de anel aromático, que está presente no espectro Raman de outras fibras como linho, juta e cânhamo; ${ }^{19}$ isso sugere que o papel do Inventário e Livro sejam feitos de fibra de algodão, que é tida como a forma mais pura de celulose $(\alpha$-celulose $) .{ }^{19}$ 
Como foi dito anteriormente, a degradação da celulose se dá principalmente pela hidrólise das ligações $\beta$-1,4-glicosídicas, que promove a ruptura da cadeia do polímero em unidades menores aumentando as regiões amorfas ${ }^{3}$ e causando mudanças na arquitetura polimérica local, com o consequente rearranjo das ligações de hidrogênio. Isso faz com que as vibrações que envolvem deformações angulares dos grupos $\mathrm{CCH}, \mathrm{COH}, \mathrm{OCH}$ e $\mathrm{HCH}$ sejam afetadas. Apesar dos espectros do papel do Inventário e do Livro serem bastante similares, no espectro FTIR a intensidade relativa da banda em $1425 \mathrm{~cm}^{-1}$ quando comparada com a banda em $1316 \mathrm{~cm}^{-1}$ (ambas deformações angulares) é diferente nos dois papéis: no caso do Inventário $\mathrm{I}^{1425} / \mathrm{I}^{1316}=0,94$ enquanto no caso do Livro esse valor é 0,59 . Esse comportamento foi atribuído por Proniewicz e col. ${ }^{3}$ à hidrólise das fibras e, provavelmente, estaria relacionado à idade do documento; não se pode descartar, entretanto, que o tipo de fibra e o processamento a que foi submetida também sejam determinantes.

Com o propósito de verificar a contribuição desses dois fatores, alguns tipos de papéis japoneses contemporâneos (Gampi, Kozo, Tengujo e Mitsumata), que divergem em relação ao tipo de fibras e de processamento da polpa, foram analisados, assim como papel sulfite comercial. A Figura 7 mostra o espectro FT-Raman dos papéis japoneses. Quando os papéis japoneses são comparados com os do Inventário e Livro é possível notar algumas diferenças, como a presença de uma banda em $c a .895 \mathrm{~cm}^{-1}$ de intensidade média, que no espectro Raman de algodão e outras fibras aparece com intensidade muito fraca. Além disso, a intensidade relativa das bandas na região entre 1200 e $1500 \mathrm{~cm}^{-1}$ é maior do que nos espectros dos papéis antigos. Esse comportamento provavelmente está relacionado às interações intra e intermoleculares por ligação de hidrogênio.

No espectro FT-Raman do papel sulfite (Figura 8) nota-se a presença de uma banda muito larga e intensa com máximo em $760 \mathrm{~cm}^{-1}$ que pode ser atribuída a hidróxido de cálcio $\left(\mathrm{Ca}(\mathrm{OH})_{2}\right)$ e também de uma banda fina e intensa em $1086 \mathrm{~cm}^{-1}$ característico de carbonato de cálcio. A presença dessas duas bandas é atribuída ao calcário

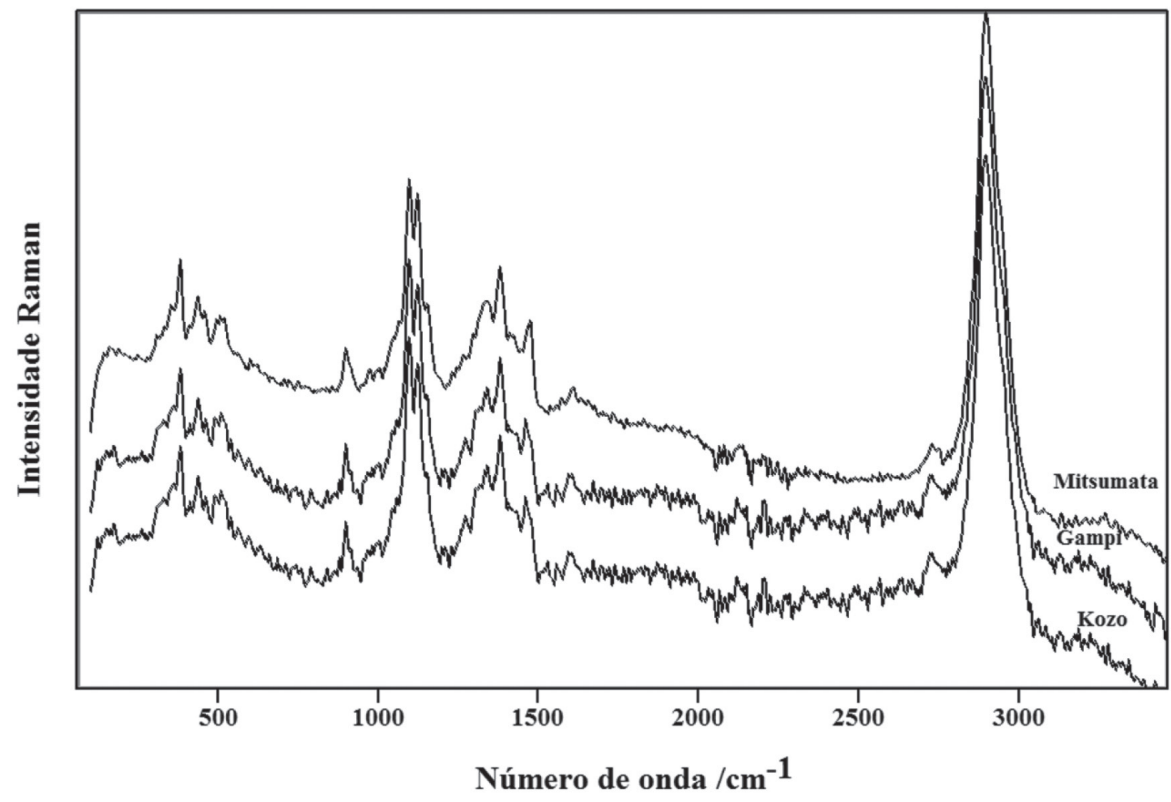

Figura 7. Espectros FT-Raman dos papéis japoneses Kozo, Gampi e Mitsumata

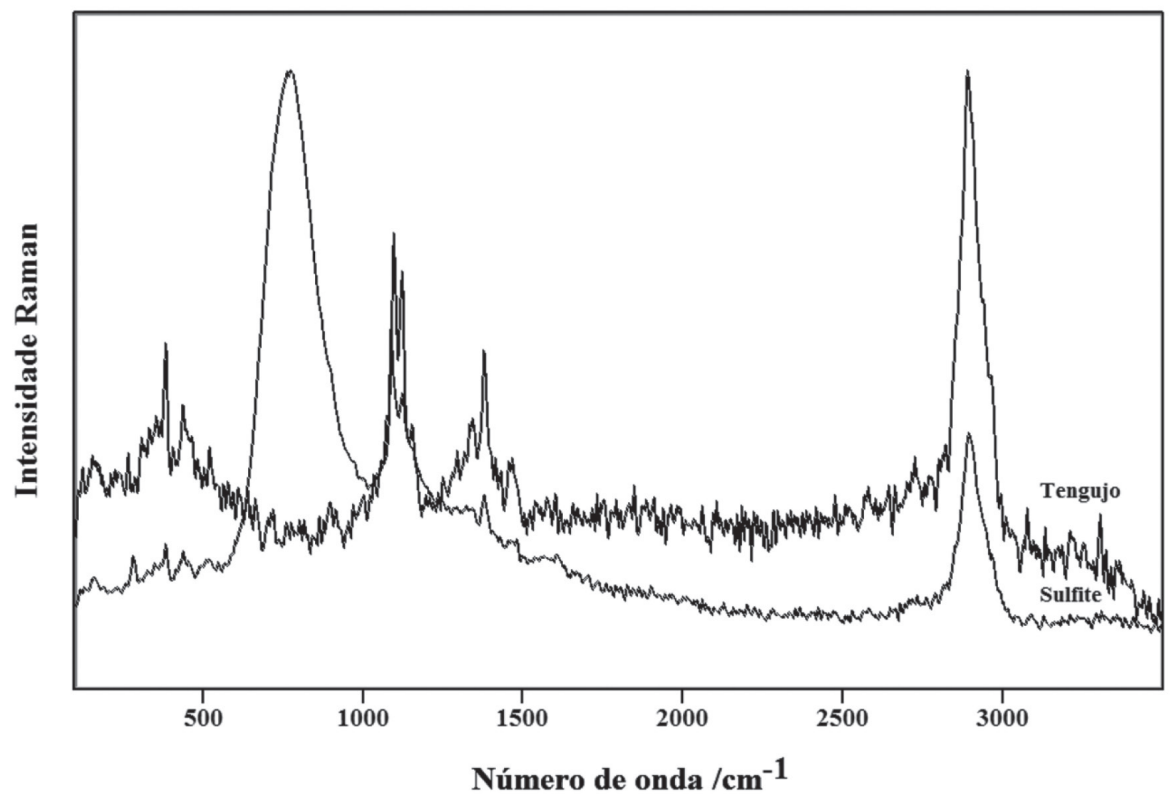

Figura 8. Espectro FT-Raman do papel japonês Tengujo e do papel sulfite 
empregado durante a extração das fibras da madeira ${ }^{20}$ (neste caso, geralmente eucalipto).

A literatura sugere que algumas variações de intensidades relativas podem ser indicativas da extensão do processo de degradação do papel. ${ }^{11-13}$ Assim, a razão das intensidades das bandas em 1121 e $1096 \mathrm{~cm}^{-1}$ atribuída por alguns autores ${ }^{3,17}$ ao estiramento simétrico e antissimétrico da ligação COC glicosídica, respectivamente, forneceria informação sobre a fissão hidrofílica das cadeias de celulose porque, de acordo com Edwards e col., ${ }^{17}$ existem variações nas intensidades relativas dessas bandas em diferentes espécimes históricos. Como foi discutido anteriormente, essas vibrações muito provavelmente não estão majoritariamente relacionadas com a ligação $\beta$-1,4-glicosídica; além disso, como os valores são próximos, não está claro se os autores efetivamente acreditam que a banda de maior número de onda corresponda ao estiramento $\mathrm{C}-\mathrm{O}-\mathrm{C}$ simétrico ou se foi um erro de revisão. De qualquer forma, esse tipo de comparação deve ser considerado com reservas pois, além das questões referentes à atribuição vibracional, as intensidades das bandas são também afetadas pelo tipo de celulose, ou seja, se são provenientes de linho, madeira, algodão ${ }^{17}$ etc., e também pelo tratamento sofrido pelas fibras durante o processo de obtenção do papel, como o demonstra os espectros obtidos dos papéis japoneses.

No caso do linho, por exemplo, Edwards e col. ${ }^{17}$ encontraram um valor de 0,76 para um linho moderno e 0,75 para um linho obtido de bandagem de uma múmia Egípcia, enquanto Alves e col. ${ }^{11}$ encontraram para um linho de uma múmia Egípcia o valor de 0,68. É interessante notar que Edwards e col. ${ }^{13} \mathrm{em}$ um trabalho sobre a análise do linho de uma das velas do "HMS Victory Trafalgar" observaram que a relação $I^{1121} / I^{1096}$ variou num intervalo de 0,64 a 0,84 o que, segundo os autores, indicaria que a quebra hidrolítica das cadeias de celulose nas ligações $\beta$-1,4-glicosídicas não é constante para diferentes partes da vela, talvez devido ao seu tamanho e a diferenças de esforço. É importante destacar, entretanto, que 0,84 é um valor substancialmente maior do que o que os autores reportam para linho moderno $(0,76)$.

Outra abordagem consiste em acompanhar o aumento na intensidade dos modos de estiramento $\mathrm{C}-\mathrm{H}$ nos espectros Raman das fibras porque a degradação leva à formação de moléculas menores. ${ }^{17}$ Dessa forma, uma diminuição no valor da razão entre as intensidades das bandas em $1096 \mathrm{~cm}^{-1}$ e $2900 \mathrm{~cm}^{-1}\left(\mathrm{I}^{1096} / \mathrm{I}^{2900}=\mathrm{R}\right)$ seria um indicador da decomposição e possivelmente da idade do papel.

Neste estudo, as intensidades foram medidas como altura das bandas, uma vez que não foi observada variação significativa no perfil das bandas avaliadas. No caso da amostra do Inventário, obteve-se para R' $\left(\mathrm{I}^{1121} / \mathrm{I}^{1096}\right)$ o valor de 0,76 e para $\mathrm{R}$ o valor de 0,55 ; já para amostras de papel do Livro R' $=0,77$ e $\mathrm{R}=0,69$; fibras de algodão novas forneceram os valores de $0,79^{17}$ e $0,71^{11}$ para $R^{\prime}$ e $0,75^{15}$ para R. Para os papéis japoneses, a partir dos espectros foram obtidos os valores de R' igual a 0,81 para o papel Gampi, 0,81 para o Kozo, 0,89 para o Tengujo e 0,92 para o Mitsumata. Analogamente, os valores de $\mathrm{R}$ foram 0,33 (Gampi), 0,33 (Kozo), 0,44 (Tengujo) e 0,27 (Mitsumata). No caso do papel sulfite, a banda em $1086 \mathrm{~cm}^{-1}$ se sobrepõe à banda da celulose em $1096 \mathrm{~cm}^{-1}$, que nesse caso está aparecendo como um ombro e, devido a isso, o cálculo da relação de intensidades R' e R fica comprometido e por esse motivo não foi incluído na Tabela 2, que sumariza os valores de R' e R encontrados para todos os tipos de papel aqui estudados. É importante destacar que no caso de $\mathrm{R}$, que usa intensidade de uma banda na região de alta frequência, a comparação com dados da literatura só tem sentido se o mesmo tipo de equipamento (dispersivo ou interferométrico) e detector (InGaAs, Ge ou InGa) for empregado por causa das diferenças que podem existir nas respostas dos instrumentos. Como podemos observar na Tabela 2 as variações de R são bem mais significativas do que R', por exemplo, quando comparamos os papéis de trapo do Inventário e do Livro, cujos valores de R são 0,55 e 0,69, respectivamente. Essa diferença pode ser atribuída a novos modos de estiramento $\mathrm{CH}$ provenientes de produtos da decomposição que aumentam a intensidade da banda em $2900 \mathrm{~cm}^{-1}$, como proposto por Edward e col. ${ }^{17}$ o que faz sentido uma vez que o papel do Inventário é mais antigo que o do Livro e se apresenta mais degradado, como pode ser visto nas imagens de SEM, Figura 9.

Tabela 2. Razões entre as intensidades de bandas, usadas no diagnóstico de estado de conservação de papéis e tecidos

\begin{tabular}{lcc}
\hline Amostra de papel & $\mathrm{R}^{\prime}=\mathrm{I}^{1121} / \mathrm{I}^{1096}$ & $\mathrm{R}=\mathrm{I}^{1096} / \mathrm{I}^{2900}$ \\
\hline Inventário (trapos) & 0,76 & 0,55 \\
Livro (trapos) & 0,77 & 0,69 \\
Mitsumata & 0,92 & 0,27 \\
Tengujo (kozo) & 0,89 & 0,44 \\
Gampi & 0,81 & 0,33 \\
Kozo-shi & 0,81 & 0,33 \\
Fibras de algodão modernas & $0,79^{17}$ & $0,75^{17}$ \\
Fibras de linho moderno & $0,76^{17}$ & $1,12^{17}$ \\
\hline
\end{tabular}

A Tabela 2 evidencia que R' não aporta informação sobre o estado de conservação das fibras porque os valores dos papéis antigos não são estatisticamente diferentes do valor para fibras de algodão moderno; já no caso de R é possível que exista uma correlação quando se consideram fibras de algodão, mas certamente é necessária uma base de dados maior a fim de que procedimentos estatísticos possam ser empregados; de qualquer forma, é importante notar que o tipo de fibra e o tratamento a que são submetidas tem papel significativo, como demonstram os valores de $\mathrm{R}$ obtidos para os papéis japoneses.

Além da caracterização por espectroscopia vibracional, empregou-se Microscopia Eletrônica de Varredura (SEM) na investigação da morfologia das fibras. A Figura 9 mostra a imagem SEM de um fragmento das fibras do papel do Inventário com ampliação de 300 vezes, em que é possível observar que as fibras são longas e compatíveis com fibras de celulose, apresentando claros sinais de degradação. ${ }^{21}$

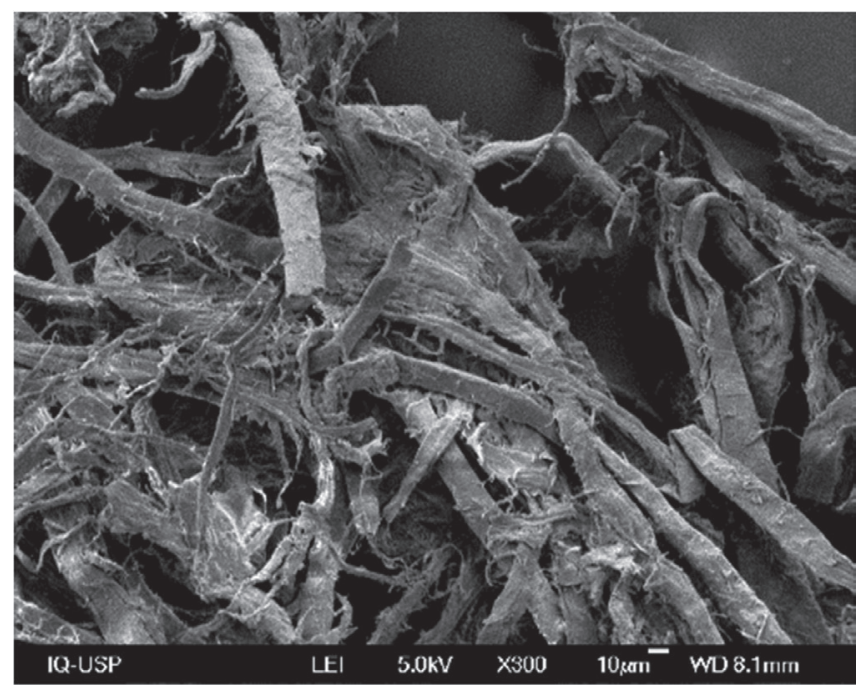

Figura 9. Imagem de micrografia SEM do papel do Inventário

A identificação colorimétrica das fibras foi feita com reagente de Herzberg aplicado sobre algumas fibras removidas do papel do Inventário; após o tingimento e secagem do reagente elas apresentavam coloração avermelhada, o que é característico de polpa de 
$\alpha$-celulose ${ }^{22,23}$ livre de lignina, tal como as fibras de algodão; o papel do Livro também apresentou o mesmo comportamento. É importante frisar, entretanto, que através desse método não é possível diferenciar fibras de algodão, linho ou cânhamo ${ }^{24}$ e, dessa forma, só foi possível concluir que se trata de papel de trapo, mas não o tipo de trapo usado.

\section{CONCLUSÕES}

Um dos objetivos iniciais do estudo do Inventário era obter informações que auxiliassem na remoção da matriz polimérica para que pudesse ser feita a restauração do documento com suporte em papel japonês. A matriz polimérica na qual o Inventário encontra-se encapsulado foi caracterizada por FTIR e por microscopia Raman, e consiste em uma camada interna de polietileno e uma externa de poli (tereftalato de etileno) (PET). Concluiu-se, porém, que não seria possível a remoção do filme de polímeros os quais haviam se infiltrado por entre as fibras do papel, sob o risco de danificação do documento. Identificou-se que o Inventário foi feito com papel de trapo, muito provavelmente celulose de algodão, e apesar da esperada degradação decorrente principalmente da hidrólise das cadeias observada pela variação na intensidade de algumas bandas nos espectros vibracionais a condição de conservação do papel é boa.

As amostras de papéis japoneses aqui investigadas apresentaram espectros Raman característicos de celulose de elevada pureza, enquanto a análise do espectro Raman de papel sulfite apresentou bandas intensas correspondendo a carbonato de cálcio devido a seu uso na desacidificação do papel.

Avaliou-se o uso das intensidades relativas das bandas Raman da celulose em 1096, 1121 e $2900 \mathrm{~cm}^{-1}$ como marcadores de envelhecimento do papel e o fato das duas primeiras vibrações estarem bastante acopladas com outros modos é uma restrição a esse procedimento. Além disso, quando se comparam diferentes tipos de papel, essas relações variam com o tipo de fibra e seu processamento, independentemente do envelhecimento; esse é o caso da comparação da celulose de papel de trapos com a celulose dos papéis japoneses. Não foi possível identificar qual material foi usado como preenchimento do papel no Livro de 1777 e no Inventário de 1578; é possível que tenha sido algum dos materiais orgânicos, como gelatina, que são citados como utilizados no período em que os papéis foram feitos. ${ }^{25}$

\section{AGRADECIMENTOS}

Os autores são gratos à Fundação de Amparo à Pesquisa do Estado de São Paulo (Fapesp) pelo apoio financeiro (processos no. 12/1311-3 e 16/21070-5). Agradecem também ao Prof. Dr. Yoshio Kawano pelas valiosas discussões sobre uso dos polímeros no processo de laminação do manuscrito e revisão cuidadosa do texto, Nathália D'Elboux Bernardino pela obtenção de espectros ATR-FTIR, Rodrigo Moreira pelo auxílio no uso do microscópio FTIR e no tingimento das fibras, Helen Ikeda pela doação do livro de 1777, Sr. Hiroyoshi Chinzei pela doação das amostras de papel japonês e à Bruker por disponibilizar o microscópio FTIR Lumos.

\section{REFERÊNCIAS}

1. Crespo, C.; La Preservación y Restauración de documentos y libros em papel: um estúdio del RAMP, UNESCO: Paris, 1984.

2. Barret, T.; Paper Througth Time, Nondestructive Analysis of $14^{\text {th }}$ through $19^{\text {th }}$ - Century Papers, http://paper.lib.uiowa.edu/european.php, acessado em outubro 2019.

3. Proniewicz, L.M.; Paluszkiewicz, C.; Weselucha-Birczynka, A.; Majcherczyk, H.; Baranski, A.; Konieczna, A., J. Mol. Struct. 2001, 596, 163.

4. Bicchieri, M.; Sodo, A.; Piantanida, G.; Coluzza, C.; J. Raman Spectrosc. 2006, 37,1186

5. Cassares, N. C.; Moe, C.; Como fazer Conservação Preventiva em Arquivos e Bibliotecas; Arquivo do Estado/Imprensa Oficial: São Paulo, 2000, vol. 5.

6. Mizumura, M.; Kubo, T.; Moriki, T.; Proceedings from the International Conference of the Icon Book and Paper Group, London, 2015.

7. Ragnar, M.; Henriksson, G.; Lindstrom, M. E.; Wimby, M.; Blechschmidet, J.; Heinemann, S.; Encyclopedia of Industrial Chemistry - Chemical Pulping Technology, Wiley-VCH Verlag GmbH \& Co. KGaA, Weinheim: 2014, cap. 1.

8. https://www.ideals.illinois.edu/handle/2142/5651. acessada em outubro 2019.

9. https://www.guiadografico.com.br/artigos/.../tecnologia-processosinsumos-substratos, acessada em outubro 2019

10. http://www.ir-spectra.com/polymers/, acessada em outubro 2019.

11. Alves, A. P. P.; Oliveira, L. P. Z.; Castro, A. A. N.; Neumann, R.; Oliveira, L. F. C.; Edwards, H. G. M.; Sant'Ana, A. C.; Vib. Spectrosc. 2016, 86, 324.

12. Teixeira, F. J.; Castro, A. A. N.; Sant'Ana, A. C.; Vib. Spectrosc. 2018, 98, 119.

13. Edwards, H. G. M.; Nikhassan, N. F.; Farwell, D. W.; Garside, P.; Wyeth, P.; J. Raman Spectrosc. 2006, 37, 1193.

14. Dauchez, M.; Langat; P.; Derreumaux, P.; Vergoten, G.; Spectrochim. Acta 1994, 50 A, 105.

15. Cael, J. J; Gardner, K. H.; Koenig, J. L.; Blackwell, J.; J. Chem. Phys. 1975, 62, 1145.

16. Backwell, J.; Vasko, P. D.; Koenig, J. L.; J. Appl. Phys. 1970, 41, 4375.

17. Edwards, H. G. M.; Farwell D. W.; Webster, D.; Spectrochim. Acta, Part A 1997, 53, 2383.

18. Makarem, M.; Lee, C. M.; Kafle, K.; Cellulose 2019, 26, 35.

19. Kavklera, K.; Demsar, A.; Spectrochim. Acta, Part A 2011, 78, 740.

20. Schimit, T.; Dariz, P.; J. Raman Spectrosc. 2015, 46, 141

21. Manso, M.; Carvalho, M. L.; Spectrochim. Acta, Part B 2009, 64, 482.

22. Jablonsky, M. L.; Dubinyová; Varga, S.; Vizarová, K.; Sima, J.; Katuscak, S. BioResources 2015, 10, 5845.

23. http://cameo.mfa.org/wiki/Graff_\%22C\%22_stain, acessada em outubro 2019

24. Konstandinovska, M.; Spirovska, Z. J.; Taylor, T.; The $4^{\text {th }}$ International Virtual Conference on Advanced Scientific Results, 2016.

25. Gorassini, A.; Calvani, P.; Baldin, A.; Mediterraneum Meeting, Italy, 2008. 